\title{
A novel technique for measuring tympanic membrane perforation size in cartilage myringoplasty
}

PYWong, E Benjamin

Department of Ear, Nose and Throat and Head and Neck Surgery, Charing Cross Hospital, London, UK.

\section{Objectives:}

In 1998, Eavey first introduced the cartilage butterfly graft technique for selected small to medium sized central perforations. This technique is performed via transcanal approach associated with minimal scarring, can be done under local anaesthetic and does not require elevation of tympanomeatal flap. The two main challenges of this technique are evaluation of the perforation size and meticulous preparation of an appropriately fitting butterfly graft. The aim of this article is to introduce a simple technique for measuring tympanic membrane perforation size to harvest the appropriately sized tragal cartilage butterfly graft.

\section{Method}

Following local anaesthetic infiltration into the tragus, the perforation is freshened and measured with a $2 \mathrm{~mm}$ Rosen round knife (Figure 1\&2).

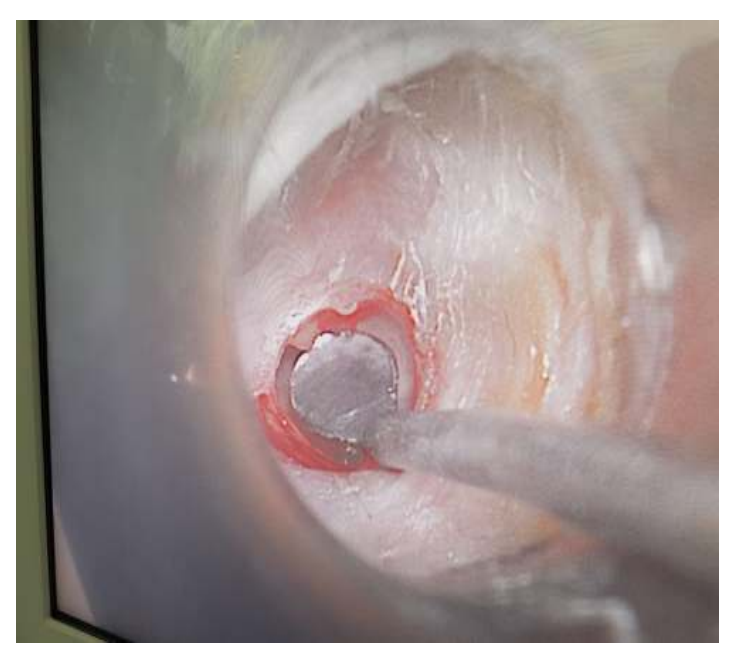

Figure 1: Measuring the perforation with Rosen round knife

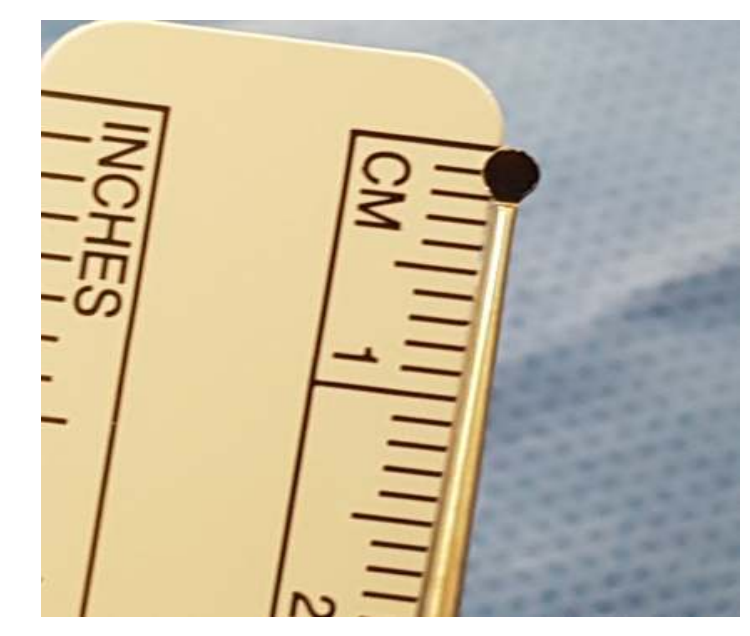

Figure 2: Diameter
Rosen round knife measures $2 \mathrm{~mm}$
Tragal incision is made and a skin punch $2 \mathrm{~mm}$ larger than the estimated perforation size is used to harvest the graft. The graft is prepared by creating a $1 \mathrm{~mm}$ deep groove circumferentially with an 11-sized blade (Figure 3).

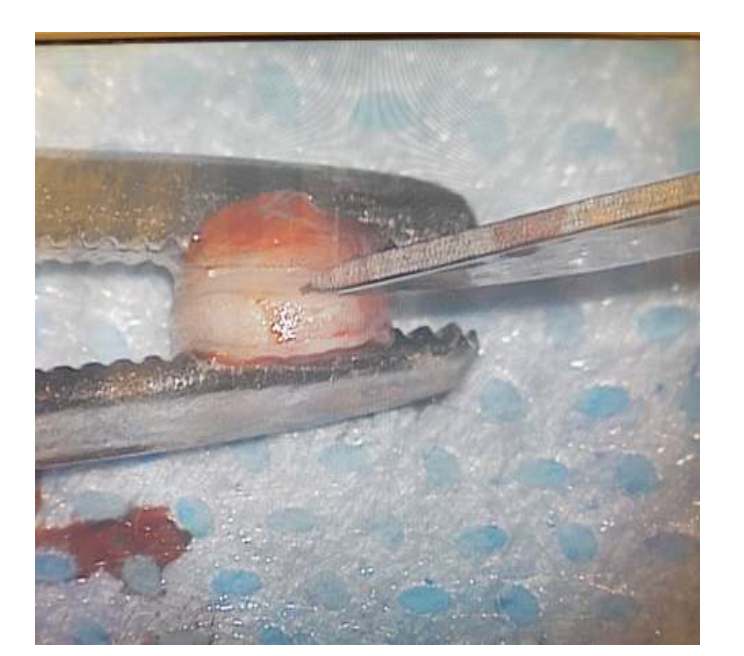

Figure 3: 11-sized blade creating the groove cirumferentially around the cartilage disc to create the butterfly graft

The groove of the graft is engaged with the anterior rim of perforation and manipulated until the perforation edges fit into the entire groove (Figure 4).

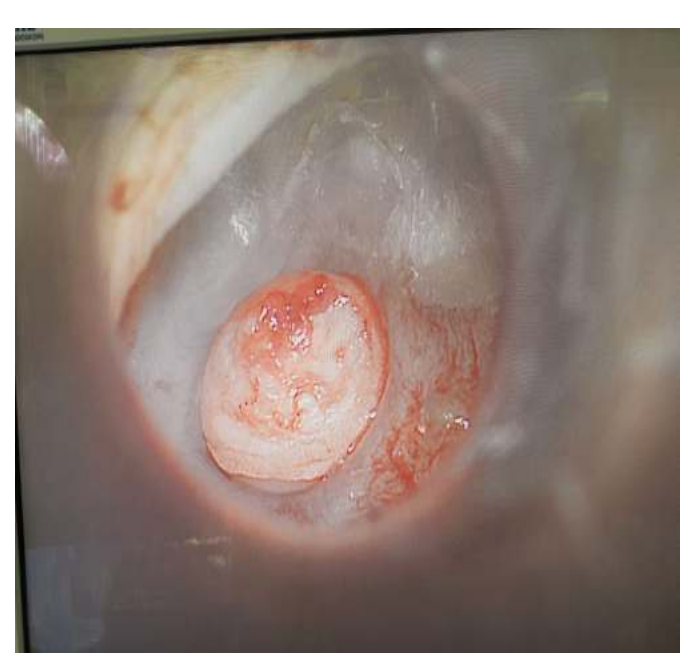

Figure 4: Butterfly graft

\section{Conclusion}

This article shown that measuring tympanic membrane perforation size with a Rosen round knife is simple, quick and easy without requiring an extra step or instrument. It provides a good size estimate for butterfly graft.

\section{References:}

1. T Rourke, J. D. Snelling, C. Aldren. Cartilage graft butterfly myringoplasty: how we do it. Clinical Otolaryngology.

2. B Eren, A Tugrul, B Ozucer, R Dogan, O Ozturan. A randomized prospective trial of a novel device for measuring perforation size during inlay 'butterfly' myringoplasty. 